\title{
A Clínica da Intersexualidade e Seus Desafios para os Profissionais de Saúde
}

The intersexuality clinic and Its challenges for health professionals

\section{Moara de Medeiros Rocha Santos \\ Psicóloga. Mestre em Psicologia. Doutoranda \\ do Curso de Pós- graduação na Universidade de Brasilia. Bolsista da Coordenação de Aperfeiçoamento de Pessoal de Nível superior (CAPES)}

\section{Tereza Cristina Cavalcanti Ferreira de Araujo

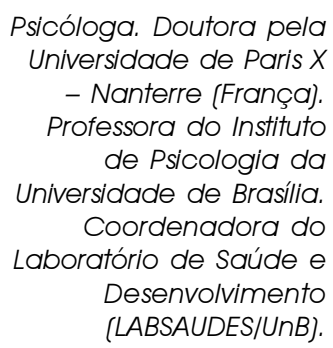

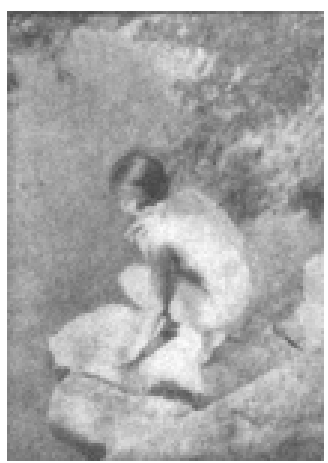

Resumo: A partir de uma revisão crítica da literatura sobre a intersexualidade, discutem-se as abordagens clínicas adotadas em casos de ambigüidade da genitália externa e/ou interna, diagnosticados como pseudohermafroditismo masculino, pseudo-hermafroditismo feminino e hermafroditismo verdadeiro. Tendo em vista a necessidade de fundamentação nas questões concernentes ao desenvolvimento psicológico, em especial a aquisição da identidade de gênero e o desempenho do papel de gênero, são enfatizadas as dificuldades envolvidas na tomada de decisão terapêutica e acompanhamento pelos profissionais que integram a equipe de saúde. Visando fornecer sugestões para futuros trabalhos nos planos científico e assistencial, é traçado um panorama das recentes pesquisas.

Palavras-Chave: Intersexualidade, hermafroditismo, clínica, profissionais de saúde.

Abstract:Based on a critical literature review on intersexuality, clinical approaches adopted in cases of external and/or internal genitalia ambiguity are discussed. These cases may have been diagnosed as male pseudo-hermaphroditism, female pseudo-hermaphroditism or true hermaphroditism. Considering the need to get support from issues related to psychological development, specifically dealing with the acquisition of gender identity and performance of gender role, the difficulties involved in the therapeutic decisionmaking and follow-up by professionals within the health team are emphasized. An overview of recent research is traced, intended to give suggestions for future scientific and care providing endeavors.

Key Words: Intersexuality, hermaphroditism, clinic, health professionals.

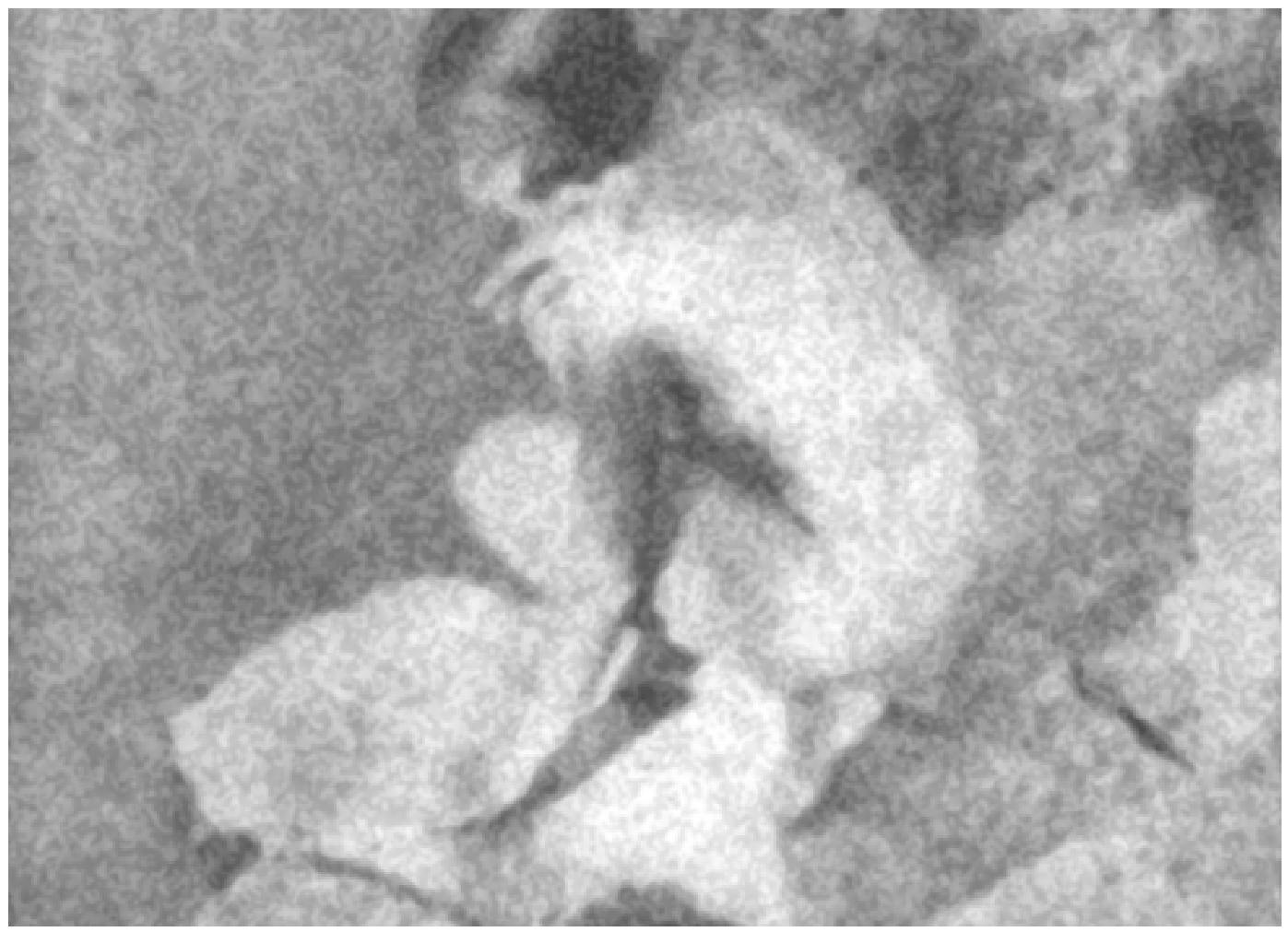

\section{Aspectos Biomédicos}

Uma cuidadosa revisão da literatura revela que o termo intersexo tem sido empregado em estudos pautados pelo interesse em aspectos psicossociais, ao passo que a denominação genitália ambígua é peculiar ao vocabulário médico em geral. De um modo ou de outro, é importante explicitar que os casos específicos diagnosticados como hermafroditismo verdadeiro, pseudo-hermafroditismo masculino e 
pseudo-hermafroditismo feminino pertencem à classificação do desenvolvimento sexual anormal. Vale lembrar que o sexo designado ao nascimento será validado (ou não) por um conjunto de características orgânicas e psicológicas (sexo de criação, identidade e papel de gênero), resultante da organização dos diferentes níveis de distinção sexual: genética, nuclear, gonadal, fenotípica e psicossocial, sendo que, inicialmente, o embrião possui capacidade para evoluir tanto para o sexo masculino quanto para o feminino. Todavia, ao longo desse desenvolvimento, certas alterações podem ocorrer, originando vários graus de diferenciação sexual anormal (Moore \& Persaud, 1995).

Atualmente, as condições intersexuais são classificadas de acordo com critérios anatômicos, histológicos, citológicos e psiquiátricos. Assim, o hermafroditismo verdadeiro é uma condição muito rara, correspondendo à diferenciação gonádica incompleta, que leva à presença de tecidos ovariano e testicular reunidos no mesmo indivíduo. Em geral, a genitália externa é ambígua, com características morfológicas predominantes do tipo masculino, o que conduz à determinação do sexo como masculino. A genitália interna pode conter útero, trompas, epidídimo e ductos deferentes. Esses indivíduos são estéreis. $\mathrm{Na}$ puberdade, podem apresentar desenvolvimento das mamas e menstruação (Speroff, Glass \& Kase, 1995).

Já os casos de pseudo-hermafroditismo possuem tecido gonadal de apenas um sexo. Pseudohermafroditismo masculino corresponde aos indivíduos incompletamente masculinizados que possuem sexo genético 46, XY e natureza testicular das gônadas. Contudo, a genitália externa não está normalmente formada e, assim como a genitália interna, pode ser ambígua ou feminina. Nos casos de pseudo-hermafroditismo feminino, os sujeitos são providos de gônadas femininas, geralmente funcionais, e uma constituição cromossômica 46, $X X$. Os órgãos genitais internos são femininos, porém a genitália externa apresenta graus de masculinização. Essa é a única condição em que os indivíduos são férteis.

Para diagnosticar esses estados intersexuais, seguese usualmente um roteiro de avaliação detalhado visando conhecer desde a história pré-natal da mãe até a condição atual do paciente, o que inclui: anamnese, exames laboratoriais da cromatina sexual, cariótipo, dosagens hormonais sangüíneas, urina (para detecção de hormônios adrenais e gonadais) e identificação anatômica dos genitais internos (através de exame ou cirurgia da cavidade abdominal). O tratamento clínico consta de terapêutica hormonal, através de estimulação androgênica (testosterona), para verificar a sensibilidade peniana, nos casos de atribuição do sexo masculino, ou uso de estrogênio e progesterona, quando o sexo final atribuído for feminino (Biazzoto,1995, Speroff, Glass \& Kase, 1995). Nos casos indicados, o tratamento cirúrgico tem como objetivos: a) tornar a genitália externa o mais funcional e semelhante quanto possível à genitália do sexo que foi definido e b) remover estruturas remanescentes do sexo oposto, para que não venham a interferir anatômica, funcional ou psicologicamente no sexo adotado ${ }^{1}$.

\section{As Teorias da Intersexualidade}

Existem, atualmente, duas abordagens predominantes destinadas ao manejo clínico da intersexualidade. A Teoria da Neutralidade Psicossexual ao Nascimento, proposta inicialmente por John Money, J.G. Hampson e J.L.Hampson, acha-se fundamentada em dois postulados: os indivíduos são psicossexualmente neutros ao nascimento e um desenvolvimento psicológico e sexual saudável depende da aparência da genitália, ou seja, para tais pesquisadores, a definição de gênero resulta essencialmente das influências do ambiente social, sendo de menor importância a contribuição dos fatores biológicos e pré-natais (Money, Hampson \& Hampson, 1955). Vale assinalar que a interação entre as influências ambiental e biológica foi reconhecida, mas não integrada teoricamente por Money, em 1975.

Os trabalhos dos adeptos dessa corrente consideram, portanto, que a educação de uma criança com condição física de intersexo, criada sem ambigüidade em relação ao sexo designado, é mais relevante para o desenvolvimento da identidade de gênero que seu sexo cromossômico. Dessa forma, se a criação for consistente com o sexo designado, nenhuma confusão de identidade/ papel de gênero seria encontrada (Rosenwald, conforme citado em Hurtig, Radhakrishnan, Reyes \& Rosenthal, 1983, Zucker, Bradley \& Hughes, 1987, Slijper, Drop, Molenaar \& de Muinck KeizerSchrama, 1998).

Tal abordagem sugere que a designação sexual seja efetuada o mais rápido possível, preferencialmente antes dos 24 meses de idade, período no qual a identidade de gênero ainda seria inconstante e passível de alteração. Após esse período de consolidação, qualquer modificação poderia acarretar o surgimento de Desordem de Identidade de Gênero e outros distúrbios psicopatológicos (Slijper, 1998, Braz, Donda, Sobreiro, Monteiro, Marques \& Mustachi, 1991). Em relação ao diagnóstico de desordem de identidade de gênero, é importante salientar que foram introduzidos novos critérios diagnósticos no DSM-IV. Assim,
1 A masculinização da genitália externa é feita em três etapas: 19) realizada entre dois $e$ quatro anos de vida compreende a remoção do conteúdo ovariano, cirurgia para liberação do testículo retido na cavidade abdominal $e$ correção do canal uretral localizado ao longo da superficie do penis; $2^{a}$ ) durante o período pré-escolar, realiza-se nova cirurgia plástica da uretra e 3a) no final da puberdade, é introduzida a prótese testicular (diante da ausência dos testículos). A feminilização da genitália é planejada em dois tempos: 10) remove-se o conteúdo testicular e faz-se cirurgia plástica do clitóris e da vulva; $2^{\circ}$ ) no final da puberdade, torna-se necessária a complementação da genitália, sendo realizada dilatação vaginal e cirurgia plástica da vagina, nos casos de vagina rudimentar ou ausência da mesma Longui \& Chiara,1997). 
quando se refere à criança, o critério A especifica, como comportamentos de identificação de gênero cruzado: o desejo de ser de outro sexo, vontade de se vestir de acordo com o outro sexo, assumir papéis estereotipados como do outro sexo, brincar com brinquedos contra-estereotipados e preferência por se envolver em atividades com pares do outro sexo. Em adultos, são considerados os sentimentos e crenças relatados pelo indivíduo. $\mathrm{O}$ critério $B$ refere-se aos sentimentos de desconforto com relação ao seu próprio sexo, incluindo fatores anatômicos ou aversão por atividades ligadas ao mesmo sexo e/ou vestir-se de acordo com o estereótipo correspondente ao seu sexo (Bradley \& Zucker, 1997).

Desse modo, o enfoque dado pela Teoria da Neutralidade Psicossexual ao Nascimento busca evitar as dificuldades dos pais em lidar com as incertezas da ambigüidade sexual de uma criança (Meyers-Seifer \& Charest, 1982). Além disso, a precoce cirurgia corretiva da genitália seria justificada, também, pela suposição de que a percepção da criança em relação ao próprio corpo é outro fator decisivo para a consolidação da identidade de gênero.

Todavia, para alguns autores, a complexidade dessa problemática exige mais do que a designação sexual ao nascimento, uma vez que a anormalidade da genitália é claramente evidente para os indivíduos e seu meio social. As próprias experiências vivenciadas com as constantes consultas médicas, procedimentos cirúrgicos e aparência física contribuem para a autopercepção de uma diferença (Zucker, Bradley \& Hughes, 1987, Hurtig, 1992).

Uma análise criteriosa denota que tal proposta de caráter intervencionista vem sendo adotada em Medicina a partir de um referencial que se pretende calcar em contribuições da Psicologia, mas cujo substrato revela-se pouco aprofundado. Justificada pelo interesse preventivo, a cirurgia realizada nos primeiros anos de vida parece responder aos impasses da conduta médica. Em outras palavras, quando se enfatiza a urgência operatória, transmite-se a idéia de que existem riscos para a saúde da criança, podendo ser este um fator que confunde a família, pois, na realidade, é raro existir tal condição. Na maior parte dos casos, a decisão pode ser adiada do ponto de vista médico.

É possível notar, então, que a ampla utilização dessa abordagem se deve, em parte, à forma decisiva e inquestionável na qual a designação sexual é feita, o que dá a impressão de que o sexo natural e verdadeiro do bebê foi finalmente descoberto e o problema está sendo enfrentado (mas, quase sempre, serão necessárias várias etapas cirúrgicas ao longo dos anos). A intervenção profissional em tal contexto de espera, dúvida e ansiedade provê alívio aos pais quanto ao sexo no qual criar a criança, endossando a proposta adotada pelo profissional médico, além de gratificar a equipe de saúde por ter condições de oferecer algum conforto para a família. Mas isso não significa que os desafios tenham sido efetivamente assumidos. Nos últimos trinta anos, a perspectiva defendida por Money vem sendo criticada em razão de constatações decorrentes de experiências profissionais e de relatos de indivíduos intersexuados de diferentes culturas. Assim, Milton Diamond propõe a Teoria da Tendência Interacionista após o Nascimento, a partir da qual existiria uma predisposição ou tendência inata que favorece o desenvolvimento da sexualidade do indivíduo em sua interação com o mundo, ou seja, tal enfoque enfatiza a interação entre forças inatas e ambientais na formação da identidade. Supõe-se que os indivíduos não são psicossexualmente neutros ao nascimento, mas, sim, predispostos a interagir com forças ambientais, familiares e sociais de um modo masculino ou feminino. Seus adeptos criticam a ênfase nos primeiros dois anos de vida e se preocupam com as repercussões clínicas da adoção irrefletida de condutas baseadas na proposta de Money (Diamond, 1965/1996-a,b, Diamond \& Sigmundson, 1997-a).

A proposta de Diamond chama a atenção para aspectos importantes do acompanhamento clínico, como a comunicação da condição intersexual aos pais e à criança. Destaca-se a necessidade de adequar a informação ao momento do desenvolvimento da criança, visando à sua participação na tomada de decisão quanto ao tratamento, o que em geral exige o adiamento da cirurgia estética até que o sujeito possa adquirir uma compreensão sobre a sua condição (Diamond, 1996 a-b, Reiner, 1997, Diamond \& Sigmundson, 1997-b).

Segundo seus adeptos, as decisões sobre a redesignação sexual não devem estar apoiadas predominantemente no prognóstico anatômico ou em um adequado funcionamento sexual (do ponto de vista anatômico/funcional), mas, sim, no desenvolvimento psicológico do sujeito. Não aconselham a cirurgia com indicação estética por pensarem que uma aparência diferenciada da genitália na criança acarreta prejuízos menores (uma vez que existem outros fatores que influenciam na construção da identidade de gênero), do que uma genitália adulta, aparentemente normal, porém, com funcionalidade comprometida e sensibilidade erótica reduzida. Por isso, argumentam que, somente após a puberdade, o paciente é capaz de informar e consentir a respeito do que fazer com seu corpo. 
Alertam, ainda, que a maioria das condições de intersexo podem permanecer sem qualquer cirurgia (Diamond \& Sigmundson,1997-b). Consideram, também, que os procedimentos defendidos pelo modelo da Teoria da Neutralidade Psicossexual ao Nascimento negam ao indivíduo a opção de escolha da própria identidade e papel de gênero. Apóiam tal posição em relatos de indivíduos adultos, os quais reclamam que deveria ter-lhes sido dado o direito de opinar sobre o tipo e a extensão da cirurgia corretiva e que não deveriam ter sido automaticamente designados para um sexo em razão da adequação e potencialidade funcional do falo. Sugerem, ainda, que os médicos considerem relevante a futura preferência sexual da criança e evitem a realização de qualquer cirurgia irreversível (Diamond 1982, 1995/1996-a).

Em síntese, é necessário reconhecer que as duas propostas para o manejo da intersexualidade, apesar de aparentemente antagônicas, estão focalizadas na decisão de quando intervir para a mudança de sexo. Do ponto de vista clínico, parece redutor o caráter prescritível de ambas. Tal característica comum pode ser explicada por terem sido geradas para atender às demandas originadas na esfera assistencial e, portanto, destinadas a auxiliar os profissionais de saúde em sua conduta. Nesse sentido, tanto uma quanto a outra parecem limitadas quanto à discussão da identidade de gênero, tal como vem sendo objeto de estudo por parte das teorias do desenvolvimento humano em Psicologia. Compreender os fatores que influenciam a escolha de uma identidade masculina ou feminina, identificando determinantes biológicos e ambientais sobre o comportamento sexual e o gênero, permanece uma importante agenda de trabalho para os pesquisadores em Psicologia (Hurtig,1992).

\section{Atuação Profissional}

Diamond e Sigmundson (1997-b), em seu roteiro para procedimentos com pessoas com genitália ambígua, afirmam que "temos que ser 'autoridades' em prover informação e aconselhamento...e não ser 'autoritários' nas nossas ações. Devemos permitir ao paciente pós-púbere um tempo para considerar, refletir, discutir e avaliar e, só então, ter a última palavra na modificação de sua genitália, papel de gênero e designação sexual final" (p. 1050).

A assistência aos casos de intersexo requer uma atuação desde o plano de diagnóstico e tratamento até um acompanhamento ao longo do desenvolvimento no ciclo vital, sobretudo no que diz respeito à dimensão psicossocial. Assim, de acordo com o desenvolvimento da criança, é possível iniciar sessões de aconselhamento com discussões sobre a sexualidade associadas à condição intersexuada (presença ou ausência de menstruação, fertilidade ou infertilidade, adoção, contracepção, opções de orientação sexual, vida marital). Durante a puberdade, devem ser apresentadas as opções cirúrgicas e endocrinológicas envolvendo cirurgia corretiva e a mudança de gênero. Se a mudança de gênero for considerada, Diamond e seus seguidores recomendam que o paciente faça um teste, vivendo por algum tempo de acordo com o sexo almejado, o que possibilitaria um primeiro ajustamento e a construção progressiva de outro papel social.

O apoio psicológico deve ser extensivo a toda a família, refletindo-se na aceitação e enfrentamento da criança frente à sua condição. Em relação aos pais, o apoio psicológico auxilia-os a solidificar o gênero designado de seus bebês, evitando que tenham uma percepção ambígua. É importante que os pais sejam consistentes com o sexo no qual a criança está sendo criada - menino ou menina além de serem congruentes com a escolha de brinquedos, jogos, amizades e aspirações futuras. Nesse sentido, é necessário que as informações Ihes sejam transmitidas de maneira detalhada e adaptada a cada momento particular do ciclo de desenvolvimento individual e familiar (ao nascimento, novamente aos dois anos de idade, início da vida escolar, antes e durante as mudanças da puberdade e periodicamente durante a adolescência).

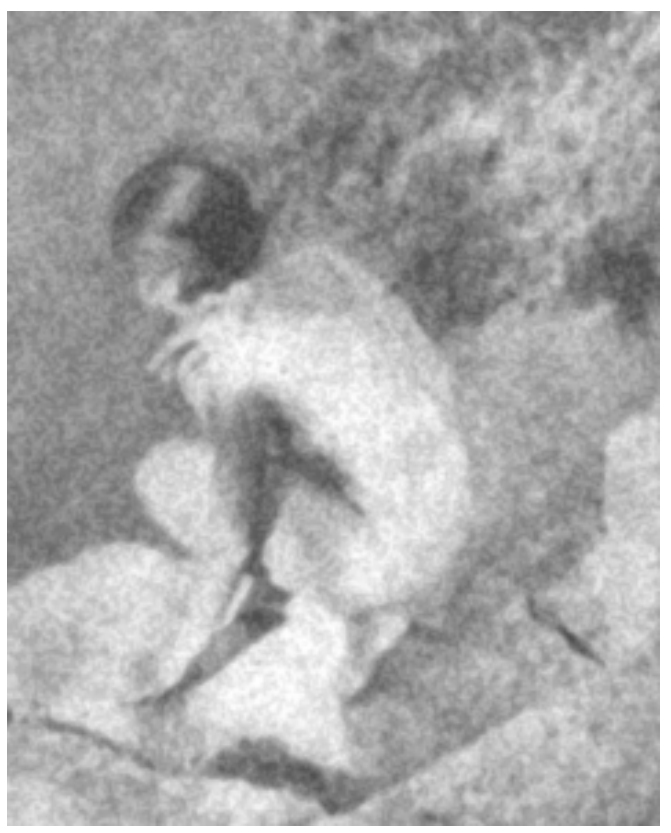

A assistência aos casos de intersexo requer uma atuação desde o plano de diagnóstico e tratamento até um acompanhamento ao longo do desenvolvimento no ciclo vital, sobretudo no que diz respeito à dimensão psicossocial.

Diante de um fenômeno complexo - que associa fatores que vão desde o nível genético até a dimensão sociocultural, além da evidente complexidade teórica e prática - é essencial tornar 
acessíveis serviços assistenciais de qualidade. Vários autores insistem na absoluta necessidade de um trabalho em equipe multiprofissional, o que indubitavelmente inclui a participação do psicólogo (Braz , 1991, Braz, Sampaio, Salvador \& Mustachi, 1984, Quattrin, Aronica \& Mazur, 1990, Pintér \& Kosztolányi, 1990). A clínica da intersexualidade não pode ser exercida apenas por profissionais da área médica. A avaliação e o aconselhamento devem ser realizados por diversos especialistas em questões de ordem sexual/gênero/ intersexo. É imprescindível um conhecimento específico em Psicologia do Desenvolvimento, sexualidade e gênero, inclusive por parte da equipe de Psicologia. Parece fundamental que, para tal desempenho, seja previsto treinamento em habilidades comunicacionais, o que se justifica não somente para atender requisitos educativos, do ponto de vista técnico/instrumental, mas sobretudo para ampliar a compreensão quanto ao significado da condição intersexual e subsidiar melhores condições interacionais na tríade sujeito-famíliaequipe.

A exemplo das experiências da América do Norte, onde se organizaram grupos de apoio - tal como o Intersex Society of North America (www.isna.org) sugere-se, tão logo seja possível, colocar a família e a criança em contato com pessoas envolvidas com a problemática, o que possibilitaria a troca de informações, sentimentos e experiências (Diamond \& Sigmundson, 1997-b). Embora essa modalidade de ajuda ainda não tenha sido implantada no Brasil, pode-se notar algum nível de mobilização associado às demandas para criação de centros especializados na clínica da intersexualidade, mas, as iniciativas ainda estão limitadas ao contexto hospitalar e provêm mais especificamente dos profissionais envolvidos com o atendimento.

\section{Pesquisas no Brasil e no Mundo}

É fundamental comentar que o delineamento dos estudos tem sido condicionado por limitações impostas pela raridade estatística da intersexualidade; a oferta de serviços de saúde especializados que possibilitaria a identificação dos participantes; além do caráter privado e íntimo vinculado às questões da sexualidade humana, sem esquecer os mais variados preconceitos que envolvem a condição intersexual, as dúvidas e comprometimentos éticos que perpassam as vivências de todos aqueles implicados.

Assim sendo, os trabalhos vêm adotando preponderantemente o método de estudo de caso. A maioria das publicações que focaliza os aspectos psicológicos resulta do acompanhamento terapêutico de um dos autores a seus pacientes. Vale ressaltar que, até hoje, são poucas as investigações voltadas para mais de um sujeito. Apesar da expectativa de que a necessidade de retorno à instituição (para acompanhamento médico e cirúrgico) favoreceria a realização de estudos longitudinais as dificuldades relacionadas à adesão ao tratamento (vergonha, privacidade, falta de perspectiva diante da repetição do tratamento cirúrgico), acabam por induzir à desistência dos participantes.

Tendo em vista a forte vinculação entre pesquisa e atuação clínica, o tema da percepção dos pais tem recebido atenção dos investigadores (Zucker, Bradley \& Hughes, 1987, Slijper, 1998, Araujo, Santos, Gay, Tamayo \& Lima, 1998, Araujo \& Lima, 1996). Assim, diante da incerteza parental quanto ao sexo do filho, um ponto crucial é entender como essa percepção da ambigüidade é transmitida para a criança, influenciando a congruência entre papel e identidade de gênero e o sexo designado. Contudo, ao se voltarem para questões relacionadas à percepção parental e práticas educativas associando-as à construção da identidade de gênero, esses estudos não têm contemplado suficientemente os aspectos comunicacionais ou interacionais, que parecem centrais na compreensão da problemática.

Dentre os tipos de intersexualidade, os casos de pseudo-hermafroditismo, tanto feminino quanto o masculino, vêm sendo mais amplamente estudados e fornecem um interessante campo de investigação quanto à influência de forças hormonais e ambientais sobre o desenvolvimento da identidade de gênero e comportamento sexual. Os casos de hermafroditismo verdadeiro, provavelmente em decorrência da sua raridade, são menos evidenciados nas pesquisas.

Existem trabalhos que têm apontado uma aparente incoerência entre o senso de masculinidade ou feminilidade e a exteriorização desses conceitos em termos de comportamento/papel de gênero em crianças com diagnóstico de pseudohermafroditismo masculino criadas como menino. Identifica-se uma identidade de gênero confusa refletida em preferências por brinquedos e/ou brincadeiras com estereótipos femininos e envolvimento em atividades socialmente determinadas para o sexo masculino (Santos \& Araujo, 1999, Dittmann, Berenbaum \& Snyder, conforme citados por Zucker, Bradley, Oliver, Blake, Fleming \& Hood, 1996). Vale lembrar que observar a criança brincando permite obter dados sobre a preferência por determinados tipos de brinquedos, o sexo da criança com quem prefere/ escolhe brincar e outros comportamentos sociais associados a atividade lúdica e ao desempenho de papéis, constituindo uma metodologia interessante para o estudo da intersexualidade 
(Hines \& Kaufman, 1994, Santos, 2000).

Na recente literatura, outro aspecto importante a ser destacado refere-se à idade na qual ocorreu a cirurgia corretiva da genitália. Para certos autores, essa variável não parece contribuir para a ambivalência em relação à identidade de gênero. Independentemente da idade na qual a cirurgia estética é realizada, o essencial, segundo eles, é oferecer orientação e mesmo acompanhamento psicológico à família e, principalmente à criança, pois através de uma abordagem terapêutica longitudinal, comprometida com o envolvimento do sujeito no tratamento, pode-se lograr uma boa adaptação do mesmo ao sexo redesignado (Hurtig, 1983, Randolph, Hune \& Rathleu, conforme citados em Hurtig, 1992, Dewhusrst \& Gordon, conforme citados por Braz, 1991, Quattrin, Aronica \& Mazur, 1990).

Em suma, um panorama geral das investigações revela que têm sido privilegiadas variáveis como identidade e papel de gênero (Santos, 2000), imagem corporal (Santos, 2000), comportamento agressivo (Hines \& Kaufman, 1994), cirurgia genital (Quattrin, Aronica \& Mazur, 1990), (re)designação sexual (Reiner, 1996, Elsayed, Al-Maghrby, Hafeiz \& Taha, 1988), desordem de identidade de gênero (Zucker, 1996, Araujo \& Santos, 1999, Santos \& Araujo, 1999), psicopatologia geral (Slijper, 1998) e desenvolvimento cognitivo ( Hurtig, 1983). As técnicas mais utilizadas para explorar tais variáveis envolvem instrumentos como: entrevistas, testes projetivos, inventários, questionários, protocolos de observação comportamental (Hines \& Kaufman, 1994) e, conforme discutido acima, os dados também vêm sendo obtidos a partir das contribuições advindas de investigações clínicas, visando sobretudo à delimitação mais precisa de critérios de intervenção próprios a tal casuística (Zavaschi, Zaslavsky, Nicilovitz \& Dorfman, 1985, Elsayed, 1988, Quattrin, Aronica \& Mazur, 1990, Reiner, 1996).

No Brasil, uma das instituições pioneiras na implantação de uma unidade de intersexo, constituída por uma equipe multiprofissional, foi o 'Hospital Infantil Darcy Vargas', em São Paulo, que, já em 1977, se propunha a estudar a intersexualidade e oferecer uma assistência médico-psicológica compatível com as necessidades dos pacientes e suas famílias, "atuando em nível preventivo, promocional, curativo e de reabilitação, nos aspectos científico, curativo, social e legal" (Braz , 1984, p. 159). Zavaschi (1985) e seus colaboradores também relatam a experiência de uma equipe de consultoria psiquiátrica do Hospital das Clínicas de Porto Alegre na condução de um caso de pseudo-hermafroditismo masculino. Atualmente, outras instituições, como o Hospital das Clínicas da Faculdade de Medicina de Ribeirão Preto da Universidade de São Paulo e o Hospital Universitário de Brasília têm se interessado pela implementação de unidades especializadas na clínica da intersexualidade.

Em linhas gerais, os trabalhos comunicados até hoje, no contexto brasileiro, têm como principal preocupação divulgar os indicadores de funcionamento dos centros de atendimento, tais como: número de casos atendidos/diagnóstico, número de intervenções cirúrgicas/ (re)designação sexual e composição e organização das equipes de saúde. Todavia, à medida que os interesses profissionais e científicos se expandem, é possível constatar uma evolução em direção a investigações mais diretamente relacionadas ao sujeito intersexuado e sua família, a exemplo do que ocorre desde a década de 50 em outros países.

\section{Considerações Finais}

No estado atual da arte, seja ela voltada para a investigação, para a clínica, ou para ambas, parece fundamental a realização de trabalhos que considerem não apenas as questões específicas vinculadas à intersexualidade, mas que se estendam também aos campos da Psicologia do Desenvolvimento e da Psicologia da Saúde, prevendo inclusive um exame mais amplo e aprofundado da associação entre saúde e gênero. Ao que tudo indica, a tendência metodológica deveria orientar-se para delineamentos que possibilitem a análise de variáveis abordadas em estudos executados em outras sub-áreas da Psicologia. Mais do que pesquisas sobre comportamentos sexualmente estereotipados, a necessidade de estudos longitudinais, centrados na qualidade de vida dos sujeitos intersexuados, permanece como meta incontornável.

No plano assistencial, freqüentemente percebida como uma doença grave e incorrigível mesmo diante das crescentes possibilidades de intervenção, a condição intersexual tem um significado essencialmente negativo para a família e para o sujeito, provavelmente suscitado pelas inúmeras dificuldades enfrentadas ao longo da sua existência. Nesses casos, o enfrentamento poderia ser compreendido semelhantemente às demais cronicidades em saúde (diabetes, hipertensão, lesão medular). No entanto, a intersexualidade se caracteriza, principalmente, como uma condição, transcendendo a associação com 'doença'. Compreender a intersexualidade por esse outro ângulo concede uma conotação favorável à adaptação integral do indivíduo e sua inserção social e faz perceber mais claramente as mudanças que ainda se fazem necessárias na esfera da atuação profissional em saúde.
No entanto, a intersexualidade se caracteriza, principalmente, como uma condição, transcendendo a associação com 'doença'. Compreender a intersexualidade por esse outro ângulo concede uma conotação favoróvel à adaptação integral do indivíduo e sua inserção social e faz perceber mais claramente as mudanças que ainda se fazem necessárias na esfera da ałuação profissional em saúde. 
Diante de constatações da literatura de que as evidências não são consistentes com a cirurgia precoce, pode-se argumentar em favor da possibilidade de adiamento. Tal atitude requer um maior comprometimento em esclarecer a situação para o sujeito, favorecendo a construção de sua identidade de gênero sem conduzir ao confronto com obstáculos intransponíveis no processo de socialização (por exemplo, na fase inicial de escolarização das crianças). Informações, obviamente adequadas à sua compreensão, são necessárias para que a pessoa possa situar-se como pertencente a um gênero, mesmo que sua aparência genital seja diferenciada.

Por fim, para que se promova a adesão do sujeito intersexuado ao seu acompanhamento, em prol de uma abordagem preventiva, há que se considerar a especificidade e complexidade de cada casuística. Isso inclui reconhecer que a orientação mais adequada pode ser operar mais cedo em certos casos. Contudo, naqueles em que as possibilidades são mais diversificadas, não se deve ficar sob o jugo de uma regra, cuja primazia é diminuir a ansiedade dos envolvidos em detrimento dos interesses do sujeito.

Contribuir para uma percepção mais adequada da experiência de ser intersexuado, redimensionando os valores (negativos) atribuídos pela sociedade, parece implicar a organização de ações para que o sujeito possa compreender sua condição e tome parte progressivamente no processo de seu acompanhamento até estar capacitado a ser mais participativo. Parece inadiável, portanto, a abordagem da qualidade de vida na clínica da intersexualidade, o que certamente implica mais desafios para seus profissionais de saúde.

\section{Contribuir para uma percepção mais adequada da experiência de ser intersexuado, \\ redimensionando os valores (negativos) atribuídos pela sociedade, parece implicar a organização de ações para que o sujeito possa \\ compreender sua condição e \\ tome parte progressivamente no \\ processo de seu acompanhamento até estar capacitado a ser mais participativo.}

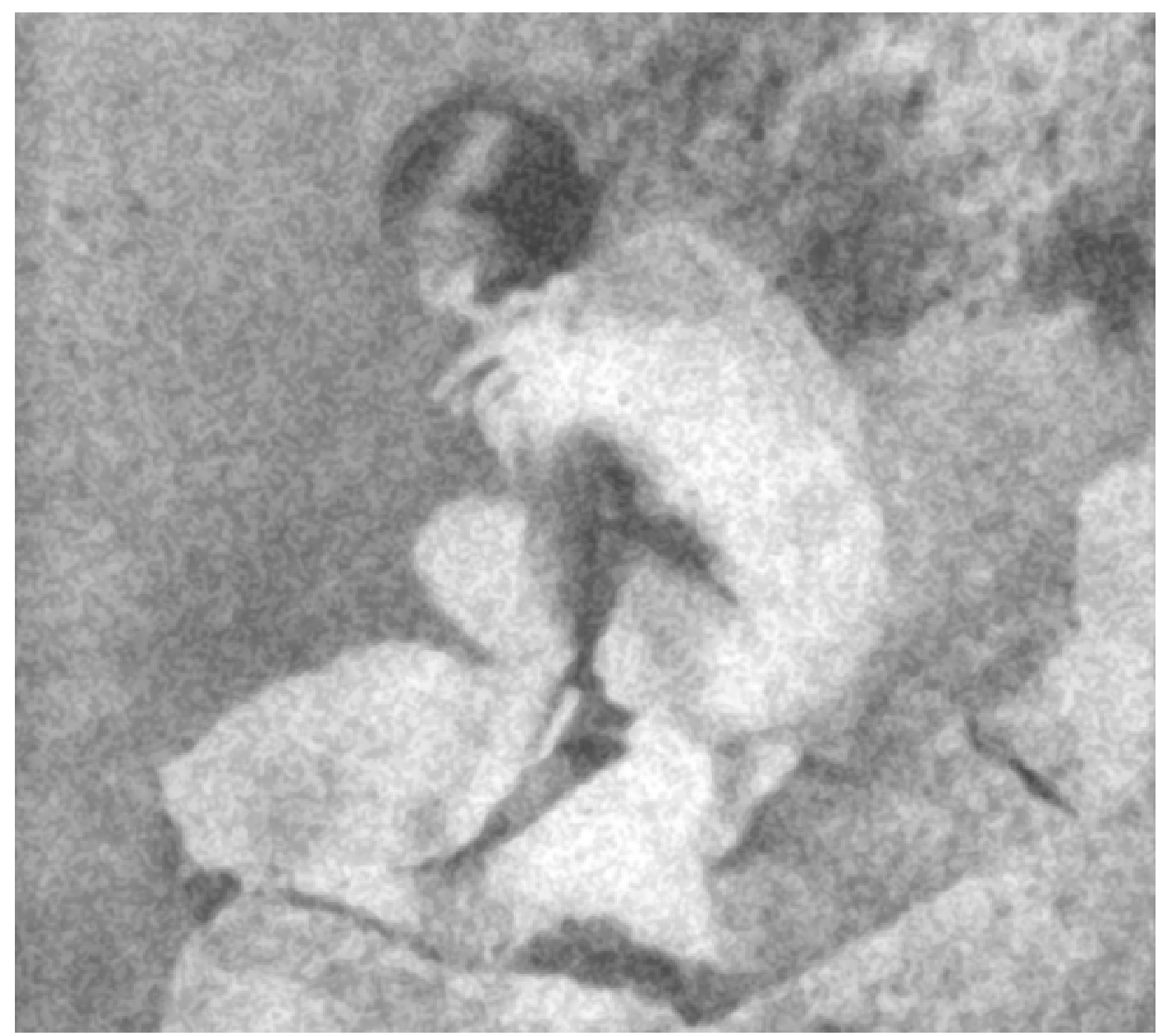


Moara de Medeiros Rocha Santos E-mail:moara@unb.br Tereza Cristina C.F. de Araújo E-mail:araujotc@unb.br Universidade de Brasília Instituto de Psicologia

ARAUJO, T. C. C. F., LIMA, A. A. M. Assistência Psicológica a Portadores de Anomalia da Diferenciação Sexual [Resumo]. In Sociedade Brasileira de Psicologia (Org.), Resumos de Comunicações Científicas, XXVI Reunião Anual de Psicologia (p.169). Ribeirão Preto: SBP, 1996.

ARAUJO, T. C. C. F., SANTOS, M.M.R., GAY, S. R.; TAMAYO, N. \& LIMA, A. A. M. Saúde e Gênero: o Atendimento Psicológico às Disfunções Intersexuais. Trabalho apresentado no II Simpósio Norte-Riograndense de Psicologia Hospitalar, Natal/RN, Brasil, 1998.

ARAUJO, T. C. C. F \& SANTOS, M. M. R. Health and Gender: Psychological Development in Cases of Intersex in the Brazilian Context [Resumo]. In M. Murray (Org). Book of Abstracts, Reconstructing Health Psychology. St.John's, Canadá: Memorial University of Newfoundland, 1999, p.45.

BIAZOTTO, J. R. Erros na Diferenciação e Desenvolvimentos Sexuais: Proposta de Roteiro Mínimo para Orientação Diagnóstica. Dissertação de Mestrado, Universidade de Brasília, Brasília, 1995.

BRADLEY, S. J. \& ZUCKER, K. Gender Identity Disorder: a Review of the Past 10 Years. Journal of American Academy of Child and Adolescent Psychiatry, 36, no 7, 1997, pp. 872-880.

BRAZ, A., SAMPAIO, D. S., SALVADOR, M. R. \& MUSTACHI, Z Organização e Funcionamento de uma Unidade de Intersexo $3 / 4$ Apresentação de 60 Casos. Pediatria Moderna, 19, no 4, 1984, pp. 151-176.

BRAZ, A., DONDA, A. C. SOBREIRO, A. R., MONTEIRO, R. C. MARQUES, S. G. \& MUSTACHI, Z. Intersexualidade 3/4 Redesignação Sexual na Adolescência com Fertilidade e Concepção. Pediatria Moderna, 27, 1991, pp. 505-519.

DIAMOND, M. Prenatal Predisposition and the Clinical Management of Some Pediatric Conditions. Journal of Sex \& Marital Therapy, 22, $\mathrm{n}$ 3, 1996-a, pp. 139-147.

Response: Considerations for Sex Assignment. Journal of Sex \& Marital Therapy, 22, no3, 1996-b, pp. 161-174.

DIAMOND, M. \& SIGMUNDSON, H. K. Sex Reassignment at Birth: Long-Term Review and Clinical Implications. Archieve of Pediatric and Adolescent Medicine, 151, 1997-a, pp. 298-304.

Management of Intersexuality: Guidelines for Dealing with Persons with Ambiguous Genitalia. Archieve of Pediatric and Adolescent Medicine, 151, 1997-b, pp. 1046-1050.

ELSAYED, S. M., AL-MAGHRABY, M., HAFEIZ, H. B. \& TAHA, S. A. Psychological Aspects of Intersex in Saudi Patients. Acta Psychiatric Scandinavia, 77, 1988, pp. 297-300.

HINES, M. \& KAUFMAN, F. R. Androgen and the Development of Human Sex-typical Behavior: Rough-and-tumble Play and Sex of Preferred Playmates in Children with Congenital Adrenal Hyperplasia (CAH). Child Development, 65, no 4, 1994, pp. 1042-1053.

HURTIG, A. L. The Psychosocial Effects of Ambiguous Genitalia. Comprehensive Therapy, 18, no 1, 1992, pp. 22-25.

HURTIG, A. L., RADHAKRISHNAN, J., REYES, H. M. \& ROSENTHAL, I. M. Psychological Evaluation of Treated Females with Virilizing Congenital Adrenal Hyperplasia. Journal of Pediatric Surgery, 18, no 6, 1983, pp. 887-893.
MEYERS-SEIFER, C. H. \& CHAREST, N. J. Seminars in Perinatology, 16 no 5, 1992, pp. 332-339.

MONEY, J., HAMPSON, J. G. \& HAMPSON, J. L. An Examination of Some Basic Concepts: The Evidence of Human Hermaphroditism. Bulletin ofJohns Hopkins Hospital, 97, 1955, pp. 301-319.

MOORE, K. L. \& PERSAUD, T. V. N. Embriologia Básica. (F.S. Vugman, Trad.) Rio de Janeiro: Guanabara Koogan, 1995.

PINTÉR, A., KOSZTOLÁNYI, G. (1990). Surgical Management of Neonates and Children with Ambiguous Genitalia. Acta Paediatrica Hungarica, 30, no 1 1, 111-121.

QUATTRIN, T., ARONICA, S. \& MAZUR, T. Management of Male Pseudohermaphroditism: a Case Report Spanning Twenty-one Years. Journal of Pediatric Psychology, 15, 1990, pp. 699-709.

REINER, W. G. Case Study: Sex Reassingment in a Teenage Girl. Journal of American Academy of Child and Adolescent Psychiatry, 35, n으, 1996, pp. 799-803.

Sex Assignment in the Neonate with Intersex or Inadequate Genitalia. Archieve of Pediatric and Adolescent Medicine, 151, 1997 pp. 1044-1045.

SANTOS, M. M. R. \& ARAUTO, T. C. C. F. Desenvolvimento Psicológico e Intersexo: um Estudo de Caso de Pseudo-hermafroditismo Masculino [Resumo]. Em Hospital Pequeno Príncipe (Org.) Temas Livres, VIII Encontro Nacional dos Psicólogos da Área Hospitalar . Curitiba: Associação Hospitalar de Proteção à Infância Raul Carneiro, 1999, p. 35.

SANTOS, M. M. R. Desenvolvimento da Identidade de Gênero em Crianças com Diagnóstico de Intersexo: Casos Específicos de Hermafroditismo Verdadeiro, Pseudo-hermafroditismo Masculino e Feminino. Dissertação de mestrado, Universidade de Brasília, Brasilia, 2000 .

SLIJPER, F.M. E., DROP, S. L. S., MOLENAAR, J. C. \& DEMUINCK KEIZERSCHRAMA, S. M. P. F. Long-term Psychological Evaluation of Intersex Children. Archives of Sexual Behavior, 27, 1998, pp. 125-144.

SPEROFF, L., GLASS, R. H. \& KASE, N. G. Endocrinologia Ginecológica Clínica e Infertilidade (H.S.A . Camargo Jr. Trad) São Paulo: Manole, 1995.

ZAVASCHI, M. L., ZASLAVSKY, J., NICILOVITZ, F. M. \& DORFMAN, M. B. Abordagem da Consultoria Psiquiátrica no Caso de uma Criança com Genitália Ambígua. Revista de Psiquiatria, 7, no 1, 1985, pp. 64-68.

ZUCKER, K. J., BRADLEY, S. J. \& HUGHES, H. Gender Dysphoria in a Child with True Hermaphroditism. Canadian Journal of Psychiatry, 32, 1987, pp. 602-609.

ZUCKER, K. J., BRADLEY, S. J., OLIVER, G., BLAKE, J., FLEMING, S. \& HOOD, J. Psychosexual Development of Women with Congenital Adrenal Hyperplasia. Hormones and Behavior, 30, 1996, pp. 300-318.
Referências 